\title{
Burnout and Commitment to Primary Care: Lessons From the Early Impacts of COVID-19 on the Workplace Stress of Primary Care Practice Teams
}

\author{
Erin L. Kelly, $P b D^{1,2}$ \\ Amy Cunningham, $P b D^{1}$ \\ Randa Sifri, $M D^{1}$ \\ Oriana Pando, $\mathrm{MPH}^{1}$ \\ Kelsey Smitb ${ }^{1}$ \\ Christine Arenson, $M D^{1,3}$ \\ 'Department of Family and Community Medi- \\ cine, Thomas Jefferson University, Philadelphia, \\ Pennsylvania \\ ${ }^{2}$ Center for Social Medicine and Humanities, \\ University of California, Los Angeles, California \\ ${ }^{3}$ Family Medicine and Community Health, \\ University of Minnesota, Minneapolis, \\ Minnesota
}

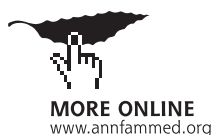

Conflicts of interest: authors report none.

\section{CORRESPONDING AUTHOR}

Erin L. Kelly

1015 Walnut Street

Curtis Building, Suite 400

Philadelphia, PA 19107

erin.kelly3@jefferson.edu

\begin{abstract}
PURPOSE The COVID-19 pandemic has dramatically affected all areas of health care. Primary care practices are on the front lines for patients seeking health care during this period. Understanding clinical and administrative staff members' strategies for managing the broad-ranging changes to primary care service delivery is important for the support of workforce well-being, burnout, and commitment to primary care.
\end{abstract}

METHODS Thirty-three staff members from 8 practices within a single health care system completed short, semistructured interviews from May 11, 2020 to July 20, 2020. Interviews were coded using a combination of conventional and directed content analysis.

RESULTS Themes emerged from the data that mapped onto the Job Demands-ControlSocial Support model. Participants reported that every aspect of primary care service delivery needed to be adapted for COVID-19, which increased their job demands significantly. Several also described pride in their development of new skills, and in most interviews, they expressed that the experience brought staff together. Staff engaged in active cognitive reframing of events during the interviews as they coped with increased workplace stress. However, as the pandemic changed from an acute stress event to a chronic stressor, staff were more likely to indicate signs of burnout.

CONCLUSIONS Primary care teams absorbed tremendous burdens during COVID-19 but also found that some stress was offset by increased support from management and colleagues, belief in their own necessity, and new development opportunities. Considering high prepandemic strain levels, the ability of primary care teams to persist under these conditions might erode as the crisis becomes an enduring challenge.

Ann Fam Med 2022;20:57-62. https://doi.org/10.1370/afm.2775.

\section{INTRODUCTION}

$\mathrm{T}$ The COVID-19 pandemic has had tectonic effects on primary care. Rapid redesign included social distancing and the use of personal protective equipment (PPE), shifting of many in-person visits to telehealth, new reimbursement models, and profound changes in scheduling, visit preparation, and patient assessment. ${ }^{1,2}$ Most COVID-19 primary care literature has focused on procedures for diagnosis and management of the disease in primary care, ${ }^{3,4}$ telehealth vs inperson utilization and differences in utilization by patient subgroups ${ }^{5.7}$ and potential health effects of treatment delays on chronic illness and cancer screening and diagnosis. ${ }^{8-10}$ To date, only a small number of qualitative studies has examined the effect of COVID-19 on primary care practice teams, all interview studies conducted early during the pandemic. One study described physicians' rapid switch to telehealth, development of new care pathways, concerns about infection, and fears about long-term patient care effects. ${ }^{11}$ Another examined workflow changes among clinicians and staff in a home-based, primary care program for older adults, ${ }^{12}$ and a third examined facilitators and barriers to primary care video visit adoption..$^{13}$ These studies provided valuable insights into changing primary care roles and workflows. However, no qualitative studies have explored the effect of COVID-19 on primary care practice team wellness. Given high burnout levels among primary care practice teams before COVID-19, ${ }^{14,15}$ understanding the effect of COVID-19 on job demands, control, support, and turnover intentions will be critical for implementing primary care workforce well-being and retention programs. The purpose of the 
present study was to explore the effect of the COVID-19 pandemic on primary care practice team wellness, burnout, and commitment to primary care.

\section{METHODS}

\section{Setting}

Thomas Jefferson Health is a large regional health system with $>90$ primary care practices affiliated with 4 campuses in southeastern Pennsylvania and southern New Jersey. Interviews were conducted from May 11, 2020 to July 20, 2020. Thomas Jefferson Health instituted a system-wide reduction of in-person visits on March 13, 2020, which triggered all practices to adopt telehealth. For context, telehealth visits at Thomas Jefferson's largest primary care practice during the first wave of the pandemic comprised $<1 \%$ of all visits in January 2020, peaked at $84 \%$ in April 2020, and comprised $33 \%$ of all visits in July 2020. Pennsylvania's first case peak was April 8, 2020, and the nadir of the first wave was June 15, 2020.

\section{Sample}

Thirty-three participants were purposively recruited to target a distribution of physicians, advanced practice clinicians, clinical care staff (medical assistants, nurses), administrative staff (schedulers, receptionists, office managers), and behavioral health consultants from the 4 Thomas Jefferson Health campuses across small to large practices. A total of 53\% (33/62) of those contacted participated.

\section{Procedures}

This study received expedited approval from the Thomas Jefferson Institutional Review Board. Staff from 8 practices at the 4 Thomas Jefferson Health campuses completed short (15-30 minute) semistructured interviews about how their practices were transformed and how workplace stress and burnout were affected by COVID-19 (Supplemental Appendix 1). Interviews were completed by $2 \mathrm{PhD}$ (E.L.K., A.C.) and $1 \mathrm{MD}$ (R.S.) faculty members from a nonparticipating primary care department. Participants were recruited during team meetings and via e-mail and provided verbal informed consent. All interviewees participated via Zoom software meetings (Zoom Video Communications Inc) from a private room at their practice or their home. Interviews were transcribed verbatim by administrative staff and a research team member (O.P.). Data were collected until the team determined saturation had been achieved. ${ }^{16}$ Participants were entered into a raffle to receive 1 of $3 \$ 100$ gift cards.

\section{Data Analysis}

Five researchers (E.L.K., A.C., R.S., K.S., O.P.) reviewed and highlighted the transcripts and identified themes. We coded and analyzed interviews using a blend of conventional and directed content analysis. ${ }^{17}$ Following recommendations of Miles and Huberman, ${ }^{18}$ we reviewed all transcripts (highlighting and making notes in the margins to identify themes), and 3 researchers (E.L.K., A.C., R.S.) collaboratively coded and iteratively developed a codebook by collectively coding 2 interviews and revising the codebook throughout. After coding 2 more interviews collectively, teams of 2 coders independently coded each transcript, and a third (E.L.K.) collaboratively reconciled coding disagreements.

After completion of the initial coding, it became clear that the themes mapped onto constructs from the Job Demands-Control-Social Support (JDCS) model, a prominent organizational psychology model (translation of the original codes to the JDCS model is presented in Figure 1). The JDCS model predicts that job strain results from the following 3 factors: job demands, control, and social support. ${ }^{19}$ Demands consist of workload, performance pressure, responsibility, hours, job complexity, role conflict, and job ambiguity. Control includes characteristics of skill discretion (opportunities for skill development and creative work), decision latitude (the ability to decide how to complete work and make decisions about work practices), and participation in decision making. Social support encompasses the feelings and behaviors that make a person feel valued and aided. ${ }^{20}$ The JDCS model suggests that social support can moderate high strain and also interacts with control. The most negative outcomes are expected among those in isolated-strain jobs (high demands + low control + low social support). ${ }^{21}$ The research team iteratively examined the relations between the codes to arrive at the central themes. Study participants had the opportunity to provide feedback as a form of member checking. ${ }^{16}$

\section{RESULTS}

Table 1 summarizes participant demographic and workplace characteristics. The sample was generally White race and female, highly experienced, and in clinical positions. Supplemental Appendix 2 lists illustrative quotes organized by JDCS model domains.

\section{Demands}

Adaptability and High Demands

Participants noted alterations to almost every aspect of practice, for example, "Literally everything has changed. From screening, phone calls, to patients coming in, to telehealth" (Office Manager). Other changes included scheduling and sanitization procedures, the elimination of physical examinations, billing rule changes, the evolving COVID-19 pandemic, protocols, role changes, redeployment, furloughs, and reduced hours. After an initial period of task reduction as in-person visits decreased, several participants reported significant increases in telephone call volume and patient telehealth sign-ups (high demands). This required patience and initiative, while at the same time they worried about patients, their own families, and themselves. 


\section{Figure 1. Mapping of initial thematic codes onto job demands-control-social support model domains, burnout, and commitment to primary care.}

\begin{tabular}{|c|c|}
\hline Original codes & Job demands-control-social support \\
\hline \multicolumn{2}{|l|}{ Demands } \\
\hline $\begin{array}{l}\text { Adaptability } \\
\text { - Changing roles and work flows } \\
\text { - Organizational response } \\
\text { Telehealth }\end{array}$ & $\begin{array}{l}\text { Demands } \\
\text { - Adaptability and increasing demands } \\
\text { - Telehealth } \\
\text { - Job security }\end{array}$ \\
\hline \multicolumn{2}{|l|}{ Job security } \\
\hline Working from home & $\begin{array}{l}\text { - Participation in decision making } \\
\text { - Redeployment }\end{array}$ \\
\hline $\begin{array}{l}\text { Communication } \\
\text { - Within practice } \\
\text { - From leadership }\end{array}$ & $\begin{array}{l}\text { - Skill development/decision latitude } \\
\text { - Working from home }\end{array}$ \\
\hline $\begin{array}{l}\text { Safety resources } \\
\text { - PPE and sanitization } \\
\text { - COVID-19 testing } \\
\text { - Hospital redeployment }\end{array}$ & $\begin{array}{l}\text { Support } \\
\text { - Practice level } \\
\text { - Organization level } \\
\text { - Safety resources }\end{array}$ \\
\hline $\begin{array}{l}\text { Mental health } \\
\text { - Fear and/or anxiety } \\
\text { - Resilience and coping }\end{array}$ & \\
\hline $\begin{array}{l}\text { Burnout } \\
\text { - Same } \\
\text { - Variable/changing over time } \\
\text { - Increasing } \\
\text { - Decreasing }\end{array}$ & $\begin{array}{l}\text { Burnout } \\
\text { - Same } \\
\text { - Variable/changing over time } \\
\text { - Increasing } \\
\text { - Decreasing }\end{array}$ \\
\hline Commitment to primary care & Commitment to primary care \\
\hline
\end{tabular}

$\mathrm{PPE}=$ personal protective equipment

access or comfort with technology, sometimes necessitated switching to telephone encounters.

Once in-person visits resumed or increased, new screening and cleaning tasks required new processes to juggle in-person and telehealth visits. Overall, most participants said their workloads had increased, fewer reported it was the same, and a couple reported reductions. In particular, the time pressure for sanitizing rooms every 20-30 minutes between appointments was a stressor for medical assistants.

\section{Job Security}

Concerns about job security were particularly acute and common, given that many practices had to reduce hours, furlough, or transfer staff, which also affected morale. A few physicians and office managers noted that they needed to reassure staff considerably, particularly in the first few weeks as patient volumes plummeted. As patient volume increased again, some participants expressed gratitude for employment.

\section{Control}

Participation in Decision Making Participants reported struggling with a sense of lost control over their work and personal lives, though individuals had different reactions to these stressors. Rapid changes made and communicated by the central organization received a

Telehealth

The greatest changes involved telehealth. Participants reported implementing expanded telehealth quickly, owing to existing infrastructure, but this required role shifts for medical assistants and other staff who had little experience with telehealth. Most physicians had performed few (if any) telehealth visits and were required to develop creative strategies, such as using cell phone lights, during physical examinations. During initial interviews, participants felt that telehealth presented serious challenges, but they acknowledged that complaints decreased as patients and staff adjusted. Participants reported pride in developing new technical skills and providing patients with mental health support, information, and reassurance. However, these new roles also increased their stress.

Participants worried about patients, particularly those of vulnerable populations, not receiving care and physical procedures. Technical issues with specific telehealth platforms, which were often ascribed to patients lacking reliable Internet mixed reception. Some were glad to have decisions made, for supplies to arrive, and for regular, high-volume communication. Others bristled regarding decisions deemed underexplained, unpredictable, or ill-suited to their practices.

\section{Redeployment}

Staff initially reported significant concern regarding redeployment to the hospital. Although ultimately hospital deployment was rare and mostly brief, it was a highly controversial possibility for clinical staff anxious about infection and job security. Among the few redeployed, some reported positive experiences, whereas others had strongly negative experiences.

\section{Skill Development and Decision Latitude}

Not all of the changes were beyond staff discretion. As noted above, some staff developed new telehealth workflows and skills, whereas others struggled with new roles. Those who returned to or remained at the office were less likely to report benefit from increased decision latitude. 


\section{Working From Home}

Before the pandemic, working from home was inconceivable. Most participants stated that working from home positively affected their work-life balance and that they remained productive. Participants found that working from home made them feel safer from infection, decreased commuting time, and provided more family time. Many welcomed new opportunities to control their schedules and procedures. For example, some behavioral health specialists were pleasantly surprised at their ability to develop therapeutic rapport, maintain high levels of referrals, and provide effective treatment. Those who had a negative experience working from home cited lack of dedicated workspace, child care, and workday structure, which created an unfavorable blending of work and personal time. Participants also cited technology barriers, including shared $\mathrm{Wi}$-FI and technical difficulties that elongated patient calls, which potentially threatened the patient-provider therapeutic alliance, along with training new employees online and patient misunderstandings about clinicians working remotely. Of the participants who mentioned working from home, 5 strongly preferred working from home, 4 disliked it, and 3 were neutral.

\section{Support}

\section{Practice-Level Support}

Most participants reported that the pandemic had increased support and camaraderie among their practice teams. In general, staff were more likely to help others, provide more frequent and higher-quality communication, and offer informal and formal support. A few participants felt that communication needed to be better within the practices, that physicians could be too critical about staff adaptations to new workflows, or that staff were becoming irritated with each other. Overall, the majority reported improvements in communication with physicians and management.

Some participants noted that they were fortunate to have behavioral health consultants to assist with the increased volume of patients reporting anxiety, stress, and depression. This ability to refer patients easily for brief mental health support or evaluation alleviated a significant care burden.

\section{Organization-Level Support}

Organizational support was perceived variably. Some participants were very grateful for all of the support offered as well as the meetings and daily e-mails, whereas others felt that the volume of information was either too much or too little. Some appreciated how limited the furloughs and hour reductions were and the measures taken to help people feel valued and protected (eg, providing adequate PPE).

\section{Safety Resources}

Safety concerns were common, and every participant recognized the importance of PPE, though they largely saw it as out of their control and constantly evolving. Two participants adamantly felt that PPE was insufficient and freely expressed

\begin{tabular}{|c|c|}
\hline Characteristic & \\
\hline Age, y, mean (SD) & 44.5 (11.9) \\
\hline \multicolumn{2}{|l|}{ Sex, No. (\%) } \\
\hline Female & $27(81.8)$ \\
\hline \multicolumn{2}{|l|}{ Race/ethnicity, No. (\%) } \\
\hline White & $27(81.8)$ \\
\hline Black & $5(15.2)$ \\
\hline Asian & $1(3.0)$ \\
\hline \multicolumn{2}{|l|}{ Role, No. $(\%)^{a}$} \\
\hline Physician & $8(24.2)$ \\
\hline Physician assistant/nurse practitioner & $3(9.1)$ \\
\hline Medical assistant/nurse & $7(21.2)$ \\
\hline Office manager & $7(21.2)$ \\
\hline Administrative staff & $4(12.1)$ \\
\hline Behavioral health consultant & $4(12.1)$ \\
\hline \multicolumn{2}{|l|}{$\begin{array}{l}\text { Thomas Jefferson Health Campus, No. } \\
(\%)\end{array}$} \\
\hline Northeast & $7(21.2)$ \\
\hline Center City & $9(27.3)$ \\
\hline Abington & $9(27.3)$ \\
\hline New Jersey & $8(24.2)$ \\
\hline Years at practice, mean (SD) & $12.5(11.9)$ \\
\hline Years in field, mean (SD) & $16.8(12.2)$ \\
\hline
\end{tabular}

a Participants could indicate that they had multiple roles, and the majority of medical assistants also had administrative roles in scheduling, billing, or reception.

their concerns. Several reported that PPE was uncomfortable. Lack of face shields and plexiglass barriers in some offices was a source of concern. Many participants stated that their PPE was sufficient. Several mentioned that more PPE will likely be used post COVID-19 during illness visits. Testing availability was not a major theme. Among the few who mentioned COVID-19 testing, other than the first week of quarantine, the testing capacity was deemed adequate or not a concern (this might reflect the low incidence of infection $[<1 \%$ among clinicians] in this hospital system).

\section{Burnout and Commitment to Primary Care}

The majority of participants reported at least some increased anxiety and stress. Feelings of burnout varied broadly, though the largest group reported that burnout did not change. A small number had fluctuations, with some noting increasing levels of burnout, several others noting decreased burnout, and a few noting variability (increasing and decreasing over time). Office managers were the most likely to discuss burnout for themselves and report it for the staff/physicians around them ( 3 of the 4 noted it increasing).

For those reporting decreased burnout, reasons included control over work hours, better work-life balance with remote work, improved communication and relationships with coworkers, and greater task variety. Those reporting 
increased burnout cited challenges with working from home, difficulties with new technologies, and increased call volume.

Respondents overwhelmingly reported a continued commitment to primary care. Reasons included feeling as if they were helping others, gratitude for job security, and the hope that primary care will become more valued. However, 1 physician assistant was already considering leaving primary care.

\section{DISCUSSION}

The influence of COVID-19 on primary care practice is ongoing. The early pandemic created heavy increases in job demands for primary care teams and massively reduced in-person visits. ${ }^{22}$ Staff in the present study appeared to be capable of managing these changes, with good attitudes despite their fears of COVID-19 infection, possible job loss, furloughs, reduced hours, and transfers. Encouragingly, few participants reported burnout, and a few were thriving while working from home. However, these results are from within the context of a health system that was largely able to procure PPE and COVID-19 tests, had a low incidence of infection $(<1 \%)$, rapidly deployed telehealth with existing tools, and had limited furloughs and layoffs. Nationally, many practices were not as well resourced and had a great deal of difficulty providing PPE and COVID-19 testing, or had little emergency cash reserves. ${ }^{23}$ Primary care physicians were at increased risk for COVID-19-related death compared to other specialties ${ }^{24}$ which greatly increased stress during the early phase of the pandemic.

The JDCS model suggests that people with high demands are resilient if also given high levels of support and control. The results of the present study suggest that primary care teams generally had high levels of demands and support with varying levels of control during the phase of the pandemic studied (staff reported limited participation in decision making but often had high levels of decision latitude as they adapted their workflows). A systematic review comparing the relative contributions of demands, control, and support to burnout found that support from supervisors and coworkers was more important than control. ${ }^{25}$ During a crisis such as COVID-19, with variable or limited control, support resources might exert an even stronger influence on well-being. Similar to prior research, ${ }^{26}$ support received from coworkers, supervisors, and from the organization was critical. Many practices reported that team cohesion increased with palpably more practice and health system support. Organizational policy decisions that prioritized staff safety and preferences increased staff members' commitment to their practices. In practices where individuals did not feel supported, there were more concerns about safety and increased stress and anxiety. In prior JDCS studies, a high level of task-based demands is the strongest predictor of burnout ${ }_{1}^{25}$ which suggests that the continued, high-intensity demands of service delivery during the pandemic are likely to overwhelm the coping resources of some primary care practice team members. It will be critical to ensure that practices provide extra resources and support and improve workflows to reduce task demands. Future studies should explore which forms of support are most useful and worthy of sustained implementation.

Greater control over decisions on how to complete one's job and on workplace practices is important for reducing the risk of burnout. Control can be difficult to achieve, however, if there are unclear job tasks (role ambiguity) or if employees receive conflicting instructions from supervisors. ${ }^{27,28}$ In the present study, owing to issues of role ambiguity, role conflict, and the need for continuous changes, control was perceived as reduced or unhelpful to offset demands. Greater opportunities for creativity and skill development are usually considered helpful aspects of control. In the present study, some participants found the opportunity to develop new skills as positive, but for others it represented another demand. This suggests that employers will need to consider the individual preferences of their practice team members. Interestingly, working from home did largely benefit those who were able to do so. Providing practice team members opportunities to have input on redesigning their positions, including the use of telehealth and working from home, could provide some critical offsets to continued high demands.

\section{Limitations}

The interviews were cross-sectional and conducted within a single health care system. Selection bias among participants might have occurred such that those who were most acutely burned out did not participate, but as reports of positive and negative experiences were common, concerns about selection bias might be somewhat mitigated.

\section{CONCLUSIONS}

Just as the COVID-19 pandemic has spotlighted preexisting challenges in our public health and health care systems and systemic gaps in health equity, it also increased awareness regarding the significant stress on primary care teams. This is an entrenched and complex issue to address. For example, recent efforts to reduce primary care physician burnout had the unfortunate byproduct of decreased well-being among other team members, ${ }^{14}$ which means that significantly more research is needed in this area if we are to create balanced methods for practice transformation. Accordingly, the JDCS model explains much of the immediate impact of the early pandemic on primary care teams, and this model can inform strategies to engage practice teams in redesigning their own jobs and work by implementing enhanced support and control strategies at the local practice and larger system levels. Evaluations developed on the basis of the JDCS framework can provide meaningful feedback to practice and system leaders to continuously improve engagement and communication strategies to provide increased but appropriate control to each team member. This provides an opportunity to learn 
from the intense lessons of the pandemic as we continue to work toward a primary care system that works for all.

Read or post commentaries in response to this article.

Key words: COVID-19; occupational stress; burnout, psychological; job demands-control-social support model

Submitted February 25, 2021; submitted, revised, September 2, 2021; accepted September 29, 2021.

Funding support: This work was funded by the Health Resources and Services Administration (HRSA) of the US Department of Health and Human Services (HHS) as part of the HRSA Primary Care Training and Enhancement: Integrating Behavioral Health and Primary Care Program (grant number TOBHP33104). The contents are those of the authors and do not necessarily represent the official views of, nor an endorsement by, HRSA, HHS, or the US Government.

Acknowledgments: We thank our participants for sharing their experiences with us.

\section{Supplemental materials}

\section{References}

1. Krist AH, DeVoe JE, Cheng A, Ehrlich $T$, Jones SM. Redesigning primary care to address the COVID-19 pandemic in the midst of the pandemic. Ann Fam Med. 2020;18(4):349-354. 10.1370/afm.2557

2. Mehrotra A, Ray K, Brockmeyer DM, Barnett ML, Bender JA. Rapidly converting to "virtual practices": outpatient care in the era of COVID-19. NEJM Catal Innov Care Deliv. 2020;1(2). 10.1056/CAT.20.0091

3. Greenhalgh T, Koh GCH, Car J. COVID-19: a remote assessment in primary care. BMJ. 2020;368:m1182. 10.1136/bmj.m1182

4. Greenhalgh T, Knight M, A'Court C, Buxton M, Husain L. Management of post-acute COVID-19 in primary care. BMJ. 2020;370:m3026. 10.1136/bmj. m3026

5. Reed ME, Huang J, Graetz I, et al. Patient characteristics associated with choosing a telemedicine visit vs office visit with the same primary care clinicians. JAMA Netw Open. 2020;3(6):e205873. 10.1001/ jamanetworkopen.2020.5873

6. Alexander GC, Tajanlangit M, Heyward J, Mansour O, Qato DM, Stafford RS. Use and content of primary care office-based vs telemedicine care visits during the COVID-19 pandemic in the US. JAMA Netw Open. 2020;3(10): e2021476. 10.1001/jamanetworkopen.2020.21476

7. Liu N, Huang R, Baldacchino $T$, et al. Telehealth for noncritical patients with chronic diseases during the COVID-19 pandemic. J Med Internet Res. 2020; 22(8):e19493. 10.2196/19493

8. Czeisler MÉ, Marynak K, Clarke KEN, et al. Delay or avoidance of medical care because of COVID-19-related concerns-United States, June 2020. MMWR Morb Mortal Wkly Rep. 2020;69(36):1250-1257. 10.15585/mmwr $\mathrm{mm} 6936 \mathrm{a} 4$

9. Jones D, Neal RD, Duffy SRG, Scott SE, Whitaker KL, Brain K. Impact of the COVID-19 pandemic on the symptomatic diagnosis of cancer: the view from primary care. Lancet Oncol. 2020;21(6):748-750. 10.1016/ S1470-2045(20)30242-4

10. Cancino RS, Su Z, Mesa R, Tomlinson GE, Wang J. The impact of COVID-19 on cancer screening: challenges and opportunities. JMIR Cancer. 2020;6(2): e21697. 10.2196/21697

11. Verhoeven V, Tsakitzidis G, Philips H, Van Royen P. Impact of the COVID-19 pandemic on the core functions of primary care: will the cure be worse than the disease? A qualitative interview study in Flemish GPs. BMJ Open. 2020; 10(6):e039674. 10.1136/bmjopen-2020-039674
12. Franzosa E, Gorbenko K, Brody AA, et al. "At home, with care": lessons from New York City home-based primary care practices managing COVID-19. J Am Geriatr Soc. 2021;69(2):300-306. 10.1111/jgs.16952

13. Artandi M, Thomas S, Shah NR, Srinivasan M. Rapid system transformation to more than $75 \%$ primary care video visits within three weeks at Stanford: response to public safety crisis during a pandemic. NEJM Catal Innov Care Deliv. 2020;1(2). 10.1056/CAT.20.0100

14. Grumbach K, Knox M, Huang B, Hammer H, Kivlahan C, Willard-Grace R. A longitudinal study of trends in burnout during primary care transformation. Ann Fam Med. 2019;17(Suppl 1):S9-S16. 10.1370/afm.26966

15. Willard-Grace R, Knox M, Huang B, Hammer H, Kivlahan C, Grumbach K. Burnout and health care workforce turnover. Ann Fam Med. 2019;17(1): 36-41. 10.1370/afm.2338

16. Morse JM. Critical analysis of strategies for determining rigor in qualitative inquiry. Qual Health Res. 2015;25(9):1212-1222. 10.1177/1049732315588501

17. Hsieh HF, Shannon SE. Three approaches to qualitative content analysis. Qual Health Res. 2005;15(9):1277-1288. 10.1177/1049732305276687

18. Miles MB, Huberman AM. Qualitative Data Analysis: An Expanded Sourcebook (2nd ed). SAGE Publications Inc; 1994.

19. Johnson JV, Hall EM. Job strain, work place social support, and cardiovascular disease: a cross-sectional study of a random sample of the Swedish working population. Am J Public Health. 1988;78(10):1336-1342. 10.2105/ ajph.78.10.1336

20. Cohen S, Wills TA. Stress, social support, and the buffering hypothesis. Psychol Bull. 1985;98(2):310-357.

21. Kelly, EL. An Examination of Public Mental Hospitals Using the Person-Environment Fit Model [Dissertation]. University of California, Irvine; 2012.

22. Heintzman J, O'Malley J, Marino M, et al. SARS-CoV-2 testing and changes in primary care services in a multistate network of community health centers during the COVID-19 pandemic. JAMA. 2020;324(14):1459-1462. 10.1001/ jama.2020.15891

23. Etz R; Advisory Group, Larry A Green Center; Primary Care Collaborative. Quick COVID-19 primary care weekly survey: series 17. Ann Fam Med. [Preprint COVID-19 Collection]. Published Jul 31, 2020. Accessed Nov 23, 2021. https://hdl.handle.net/2027.42/156060

24. Gouda D, Singh PM, Gouda P, Goudra B. An overview of health care worker reported deaths during the COVID-19 pandemic. J Am Board Fam Med. 2021; 34(Suppl):S244-S246. 10.3122/jabfm.2021.S1.200248

25. Luchman JN, González-Morales MG. Demands, control, and support: a metaanalytic review of work characteristics interrelationships. J Occup Health Psychol. 2013;18(1):37-52. 10.1037/a0030541

26. Charoensukmongkol P, Phungsoonthorn T. The effectiveness of supervisor support in lessening perceived uncertainties and emotional exhaustion of university employees during the COVID-19 crisis: the constraining role of organizational intransigence. J Gen Psychol. 2021;148(4):431-450. 10.1080/00221309.2020.1795613

27. Hobfoll SE, Freedy J. Conservation of resources: a general stress theory applied to burnout. In: Schaufeli WB, Maslach C, Marek T, eds. Series in Applied Psychology: Social Issues and Questions. Professional Burnout: Recent Developments in Theory and Research. Taylor \& Francis; 1993:115-133.

28. Boles JS, Wood JA, Johnson J. Interrelationships of role conflict, role ambiguity, and work-family conflict with different facets of job satisfaction and the moderating effects of gender. Journal of Personal Selling $\&$ Sales Management. 2003;23(2):99-113. 